\title{
The Design of the Automatic System of Workpiece Based on PLC
}

\author{
Jun $\mathrm{Ji}^{1,}{ }^{,},{ }^{*}$, Fei-Fei Xing ${ }^{2, \mathrm{~b}}$, Bing Lu ${ }^{1}$ \\ ${ }^{1}$ Beijing Polytechnic, Beijing 100176, China \\ ${ }^{2}$ BMEI CO., LTD., Beijing 100027, China \\ aji_jun2000@sina.com, ${ }^{b}$ xingfeifei@126.com
}

Keywords: Workpiece transferring; Programmable controller; PLC

Abstract. According to requirements of automatic system, this paper designs a transmission and control system for industrial workpiece. In the system, the control part is based on the touch screen module and the programmable controller; the implement part consists of pneumatic solenoid valve-cylinder pneumatic-drive unit. The system realizes whole automatic operation of the system and completes sorting of the material.

\section{Introduction}

In the 1920s, with the development of the car, rolling bearings, carefully motor and sewing machines, automatic line appeared in machinery manufacturing[1]. With the development of industrial automation control technology, automatic production line more and more is integrated into every aspect of the industry. The automatic production line includes transmission and control system that connects automatic machine tools and auxiliary equipment by the process sequence[2-3]. In mass production, automatic line can improve labor conditions, shorten the production area and cycle, reduce production costs and ensure the production balance.

\section{Composition of the automatic transmission system}

The paper's experimental platform uses YL235A integrated device. The system is composed of feeding system, conveying system and transportation and sorting system. The control system adopts the module combination, which includes touch screen module, PLC module, frequency converter module, button module, power module, terminal block and various sensors. The overall structure is shown in Fig.1.

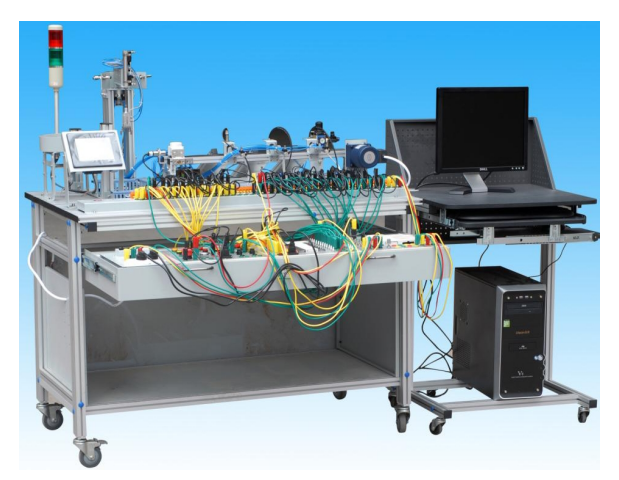

Fig.1. The overall structure

The electrical part is composed of power module, button module, PLC module and frequency converter module.Among them, the power supply module consists of three phase power supply 
switch, fuse, single phase power plug, and the connection way uses safety wire way. Button module offers a variety of different function buttons; PLC module uses SIEMENS S7-200. Fig. 2 shows the structure of the electrical part.

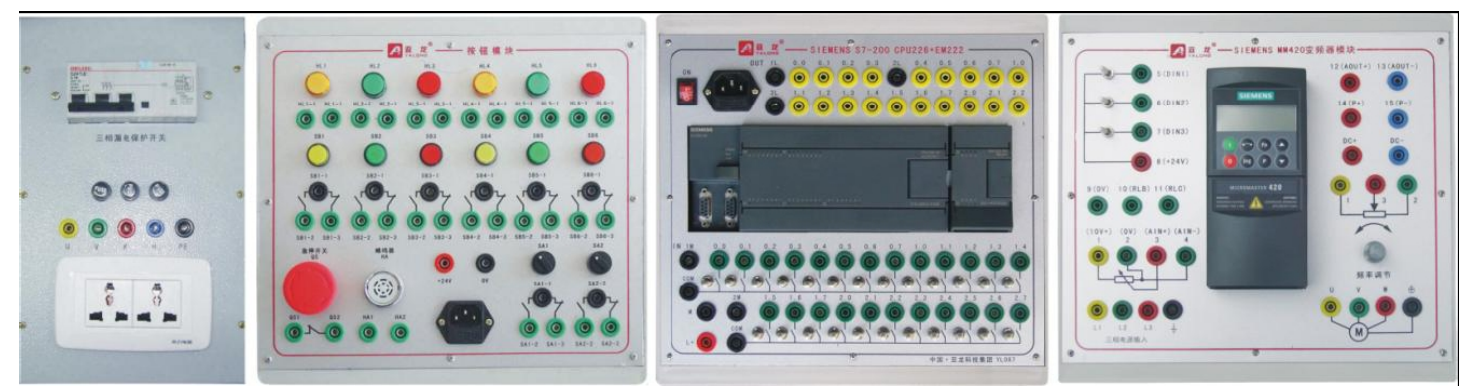

Fig.2. The structure of the electrical part

\section{System control requirements and I/O settings}

\section{Control requirements}

In production line, the workpiece should be moved from A to B by the manipulator, and the manipulator must remain in the initial position for starting. The initial position is limited on the left, hand grasping loosen, and the cantilever cylinder rising.

Firstly, turn on the power supply and press the start button SB1, when the material detection sensor detects a workpiece, the machine will perform the following actions: robot arm stretch out $\rightarrow$ arm drop $\rightarrow$ delay $1 \mathrm{~s} \rightarrow$ hand grasp to intensify the material $\rightarrow$ arm up $\rightarrow$ the cantilever is retracted $\rightarrow$ rotating machine to the right $\rightarrow$ cantilever $\rightarrow$ arm drops $\rightarrow$ delay $1 \mathrm{~s} \rightarrow$ hand grasp release, $\rightarrow$ arm up $\rightarrow$ the cantilever is retracted $\rightarrow$ rotating machine to the left. If the input material detection sensor has signals, the system will continue working; when the workpiece drops in the material input location, the sorting mechanism sorts workpiece to different material groove.

\section{I/O settings}

According to the control requirements, the I/O settings are defined as Table 1. 
Table $1 \quad$ I/O settings

\begin{tabular}{|c|c|c|c|c|c|}
\hline \multicolumn{3}{|r|}{ Input } & \multicolumn{3}{|c|}{ Output } \\
\hline SN & Address & Comments & $\mathbf{S N}$ & Address & Comments \\
\hline 1 & $\mathrm{I} 0.0$ & start & 1 & Q0.0 & Cantilever \\
\hline 2 & I0.1 & stop & 2 & Q0.1 & Cantilever recovery \\
\hline 3 & I0.2 & Rotating left limit sensor & 3 & Q0.3 & Arm drops \\
\hline 4 & I0.3 & Rotating right limit sensor & 4 & Q0.4 & Arm up \\
\hline 5 & I0.4 & Material detection sensor & 5 & Q0.5 & Hand grip \\
\hline 6 & I0.5 & Fiber optic sensor2 & 6 & Q0.6 & Hand grasp release \\
\hline 7 & I0.6 & Fiber optic sensor 1 & 7 & Q0.7 & Left turn left \\
\hline 8 & $\mathrm{I} 0.7$ & Inductance sensor & 8 & Q1.0 & Left turn right \\
\hline 9 & I1.0 & Feed mouth photoelectric sensor & 9 & Q1.1 & Push material 1 \\
\hline 10 & I1.1 & Cantilever cylinder limit & 10 & Q1.2 & Push material 2 \\
\hline 11 & $\mathrm{I} 1.2$ & Cantilever cylinder limit & 11 & Q1.3 & Push material 3 \\
\hline 12 & I1.3 & Arm cylinder cap & 12 & Q1.4 & Driving alarm \\
\hline 13 & I1.4 & Arm cylinder limit & 13 & Q1.5 & Driving frequency \\
\hline 14 & I1.5 & Hand grasp the cylinder to tighten limit & 14 & Q1.6 & Run indicator \\
\hline 15 & I1.6 & Push material cylinder 3 & 15 & Q1.7 & Stop indicator \\
\hline 16 & I1.7 & Front limit Push material cylinder 3 & & & \\
\hline 17 & $\mathrm{I} 2.0$ & Push material cylinder 2 & & & \\
\hline 18 & $\mathrm{I} 2.1$ & Front limit Push material cylinder 2 & & & \\
\hline 19 & $\mathrm{I} 2.2$ & Push material cylinder 1 & & & \\
\hline 20 & $\mathrm{I} 2.3$ & Front position of Push material cylinder 1 & & & \\
\hline
\end{tabular}

\section{Function table}

The function of the mechanical hand moving mechanism is shown in Fig.3. 


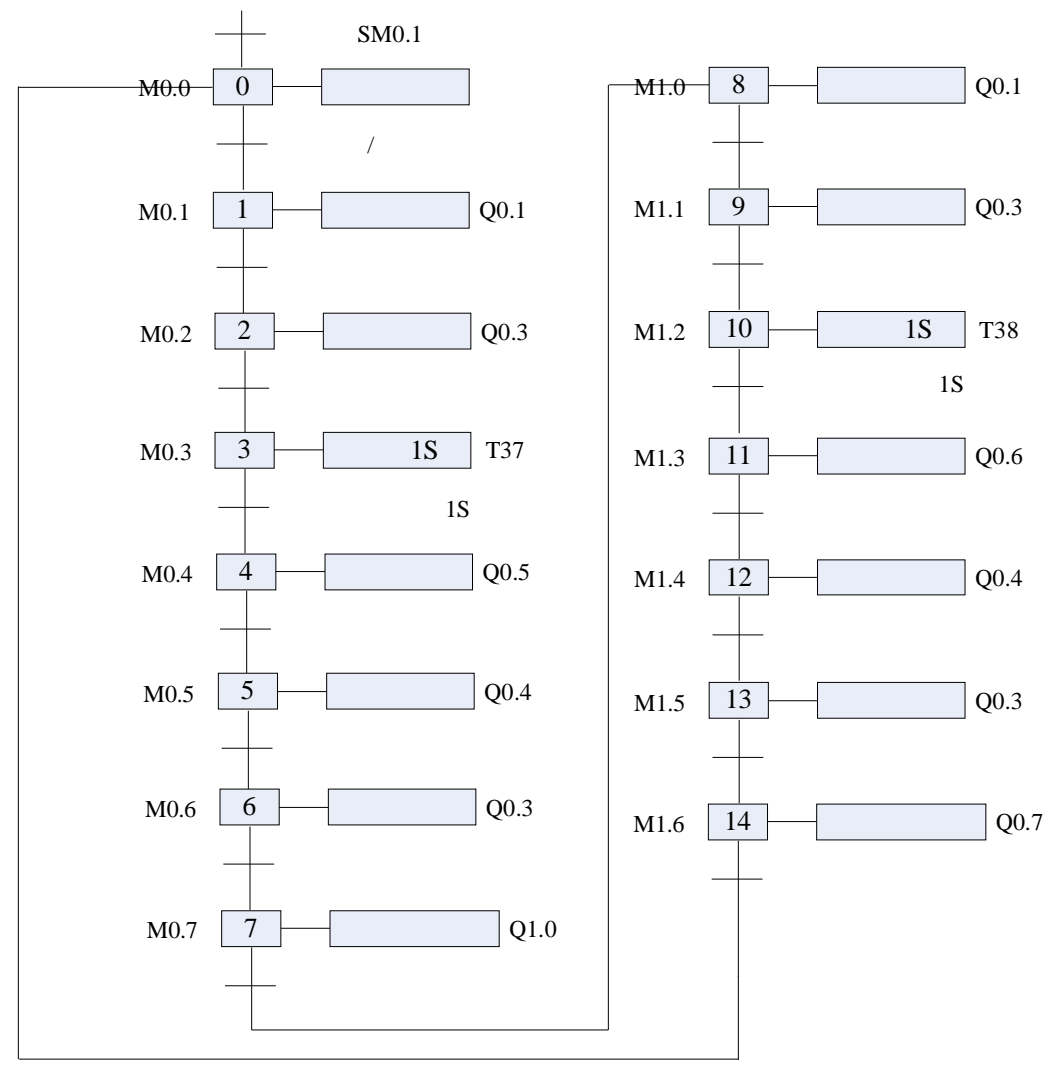

Fig.3. Function table

\section{Conclusion}

In this paper, the automatic transmission of the workpiece is realized based on the YL235A integration device. The I/O of the system is set up by analyzing the control requirements. The function of the system is designed according to the work flow.

\section{Reference}

1 A.M. Cheded. Control of a four-level elevator system using a programmable logic controller. International Journal of Electrical Engineering Education.(2003)

2 S.L. Ren. Development of PLC-based Tension Control System. Chinese Journal of Aeronautics.20:266-271 (2007)

3 Y.Y. He. A Control System of Material Handling in FMS. Journal of shanghai university. 1:1(1997) 